\title{
XÂY DỰNG CÔNG CỤ CẢI THIỆN Độ CHÍNH XÁC CỦA BẢN ĐỒ ĐỊA CHÍNH CŨ
}

\author{
TRƯƠNG SONG HÒA(1), NGUYẼ̃N MẠNH DŨNG ${ }^{(1)}$, NGUYẼ̃N VĂN HIỆP(2) \\ (1) Viện Khoa học Đo đạc và Bản đồ \\ ${ }^{(2)}$ Trung tâm Dũ liệu và Thông tin Đất đai, Tổng cục Quản lý đất đai
}

\section{Tóm tắt:}

Trong bài báo giới thiệu giải pháp nhằm cải thiện độ chính xác về không gian của bản đồ địa chính cũ đã được đo vẽ thành lập trước nhũ̃ng năm 2009 theo các quy phạm cũ. Tù việc phân tích các yếu tố tác động đến độ chính xác của bản đồ địa chính cũ so với yêu cầu hiện nay, để nhận thấy mức độ hạn chế theo tùng khu vưc đo vẽ trong mỗi đơn vị hành chính cấp xã/phường, tù đó đề ra thuật toán cải thiện, giải pháp nâng cao độ chính xác của tùng khu vục đo vẽ và của xã/phuờng nói chung dựa trên các điểm khống chế song trùng, tù đó tạo co sở cho lập trình Modul phần mềm VPAI chạy trên nền MicroStation có chức cải thiện độ chính xác không gian. Dũ liệu được cải thiện sẽ đồng nhất hơn với dũ liệu địa chính được đo vẽ trong nhũng năm gần đây, đảm bảo cho việc xây dụng CSDL đất đai. Bài báo là kết quả nghiên cúu của đề tài cấp co sở "Nghiên cúu đề xuất giải pháp kỹ thuật nhằm nâng cao hiệu quả sủ dụng của bản đồ địa chính cũ đáp ứng yêu cầu xây dụng CSDL đất đai trong giai đoạn hiện nay”.

\section{1. Đặt vấn đề}

Các địa phương trong cả nước đã và đang triển khai xây dựng cơ sở dữ liệu địa chính từ cấp xã/phường và tiến tới hoàn chính cho từng tỉnh/thành phố, dựa trên bản đồ địa chính đã được đo vẽ từ nhiều giai đoạn. Vấn đề phân tích đánh giá chất lượng các loại bản đồ địa chính về độ chính xác không gian cũng như mức độ đầy đủ và hiện thời của thông tin thuộc tính, từ đó chuẩn hóa đưa vào cơ sở dữ liệu địa chính là những yêu cầu quan trọng đối với dữ liệu địa chính nói chung và bản đồ địa chính nói riêng. Bản đồ địa chính đã được đo vẽ ở nhiều thời kỳ, với công nghệ khác nhau, với tiêu chuẩn kỹ thuật khác nhau, độ chính xác khác nhau, nhưng khi chuẩn hóa đưa vào một cơ sở dữ liệu thì phải thống nhất về chất lượng nói chung. Dữ liệu địa chính là dạng dữ liệu đặc biệt mang tính lịch sử bởi tính pháp lý của hồ sơ địa chính, luôn phải tồn tại song hành với sổ cách, giấy chứng nhận quyền sử dụng đất và các chứng thư pháp lý khác. Chúng ta không đủ kinh phí, thời gian và nhân lực để đo vẽ mới thay thế dữ liệu cũ được mà phải sử dụng chúng trong hoạt động quản lý dất đai theo pháp luật. Chính vì vậy vấn đề cải thiện độ chính xác của bản đồ địa chính cũ đang được quan tâm không những trên thế giới mà ở Việt Nam đã và đang đặt ra.

Công tác thành lập bản đồ địa chính ở nước ta đã trải qua một thời gian dài với nhiều công nghệ đo đạc khác nhau từ đơn giản đến hiện đại, nhiều quy phạm kỹ thuật khác nhau, từ hồ sơ trên giấy đến hồ sơ số hóa đã làm cho dữ liệu địa chính hiện nay có độ chính xác về không gian trong mỗi đơn vị hành chính thường không đồng nhất, ngoài ra khi chuyển từ dữ liệu giấy sang dữ liệu số cũng chứa nhiều sai số khác nhau, yêu cầu độ chính xác đối với các tỷ lệ đo vẽ cho các nhóm đất cùng khác nhau... Tất cả các nguyên nhân trên tác động đến chất lượng dữ liệu địa chính cũ so với bản đồ địa chính được đo vẽ trong những năm gần đây. Việc đánh giá chất lượng của bản đồ địa chính cũ thường dựa vào các quy định về sai số đo vẽ bản đồ, tỷ lệ bản đồ, công nghệ thành lập theo các quy phạm cũ. Đặc biệt trong giai đoạn 1991 - 2009 bản đồ địa chính được thành lập theo các quy phạm 1996 và quy phạm 2008, có chất lượng không cao và được 
lưu ở dạng giấy, sau đó được số hóa để xây dựng CSDL địa chính, vì vậy để cải thiện hiệu quả sử dụng và chất lượng của CSDL địa chính chúng ta chưa có một đánh giá chi tiết và giải pháp nhằm cải thiện chất lượng của dữ liệu cho tương thích và phù hợp với chât lượng của dữ liệu địa chính đo từ năm 2010 đến nay (Giai đoạn này quy định độ chính xác đo vẽ bản đồ địa chính cao hơn).

Nhiều quốc gia trên thế giới cũng gặp trường hợp tương tự như Việt Nam, khi xây dựng và hiện đại hóa cơ sở dữ liệu địa chính từ dữ liệu địa chính cũ. Xây dựng CSDL địa chính đều phải sử dụng dữ liệu địa chính được đo vẽ từ rất lâu trong các hồ sơ địa bạ, bằng khoán cũ. Tất cả dữ liệu này đều có độ chính xác thấp do được đo vẽ với các dụng cụ thô sơ từ những năm đầu thế kỷ 20. Chính vì vậy việc tìm kiếm giải pháp để chính xác hóa nguồn tư liệu này là cần thiết nhằm đưa dữ liệu hồ sơ địa chính vào quản lý ở dạng CSDL địa chính. Công việc này được gọi là "Cải thiện độ chính xác vị trí", là một quá trình cải thiện vị trí của tọa độ hình học của các thửa đất và các đối tượng thể hiện trên bản đồ địa chính, để phản ánh tốt hơn vị trí thực tế của nó cả về vị trí tuyệt đối và vị trí tương đối. Cải thiện độ chính xác được hiểu là việc tinh chỉnh dữ liệu có độ chính xác thấp dựa vào các điểm khống chế mặt đất có độ chính xác cao hơn. Các nghiên cứu của Felus Y.A (2007); Hesse W.J (1990); Hope S, Gordini C (2008); Frank Gielsdorf (2004); Hashim et al (2016); Nedim tuno, Admir Mulahusic, Dusan Kogoj (2017); Ronsdorf (2008); Tong X, Liang D, Xu G, Zhang S. (2011); Wolf, P., \& Ghilani, C. (2006); David Siriba (2009); Nedim Tuno. (2017) đã tập trung vào các nhóm giải pháp cải thiện độ chính xác không gian của bản đồ địa chính như lựa chọn thuật toán nắn chỉnh, lựa chọn mô hình chuyển đổi dựa trên các điểm khống chế song trùng có trên bản đồ cũ và xác định được trên thực địa hiện nay. Cải thiện độ chính xác vị trí (Positional Accuracy Improvement - $\mathrm{PAI}$ ) nhằm nâng cao độ chính xác vị trí thực tế của thửa đất cả về vị trí tuyệt đối và vị trí tương đối. PAI được hiểu là việc tinh chỉnh dữ liệu có độ chính xác thấp dựa vào dữ liệu có độ chính xác cao hơn. Hình 1 minh hoạ khái niệm PAI.

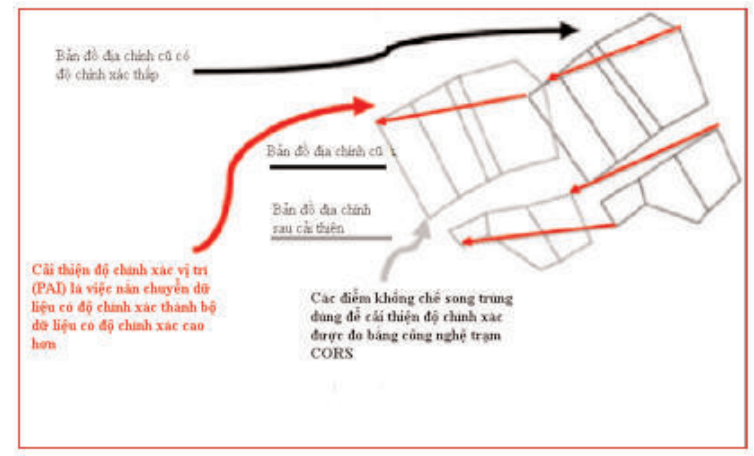

Hình 1: Khái niệm cải thiện

độ chính xác vị trí (PAI)

Cải thiện độ chính xác vị trí đối với dữ liệu địa chính cũ là một vấn đề đã được nhiều quốc gia thực hiện trong quá trình số hóa địa chính tiến tới xây dựng CSDL đất đai. Xây dựng được bộ dữ liệu bản đồ địa chính của mỗi quốc gia thường trải qua thời gian dài, với sự hao tốn về kinh phí, nguồn lực, thiết bị và công nghệ, do vậy khi xây dựng CSDL không thể không sử dụng các tư liệu địa chính cũ để chuyển đổi từ bản đồ giấy sang bản đồ số và quản lý ở dạng CSDL là điều tất yếu của nhiều quốc gia. Việt Nam cũng nằm trong xu thế trên, từ trước những năm 2000, nhiều địa phương còn thành lập bản đồ địa chính gốc trên giấy, theo quy phạm địa cũ, yêu cầu độ chính xác thấp hơn hiện nay, công nghệ thiết bị ở mức độ trung bình... đã tạo ra bộ dữ liệu cũ có độ chính xác không cao so với dữ liệu địa chính được đo vẽ trong những năm gần đây, đã tạo khó khăn trong việc đánh giá tư liệu cũ trong xây dựng CSDL địa chính. Chính vì vậy việc nghiên cứu tạo ra công cụ phần mềm hỗ trợ cải thiện độ chính xác của bản đồ địa chính cũ là việc làm có ý nghĩa thực tiễn cao.

\section{Giải quyết vấn đề}

2.1. Co' sở phuơng pháp luận cho việc cải thiện độ chính xác của dũ liệu địa chính cũ.

- Độ chính xác của dữ liệu địa chính của một xã/phường là không đồng nhất bởi các lý do sau: tỷ lệ đo vẽ khác nhau cho các loại đất khác nhau, công nghệ đo vẽ khác nhau và ở những thời điểm 
khác nhau, chất lượng số hóa và nắn bản đồ giấy, quy định của các văn bản quy phạm theo các thời kỳ khác nhau, hệ tọa độ hay cơ sở toán học...sẽ tạo ra các khối địa chính trong một xã/phường có độ chính xác khác nhau và chất lượng chưa tương ứng như dữ liệu địa chính hiện nay.

- Khi gộp dữ liệu bản đồ địa chính cũ phục vụ xây dựng CSDL địa chính, cần thiết phải kiểm tra độ chính xác về không gian, thu thập đầy đủ các thông tin về quá trình thành lập (Metadata), từ đó sẽ đánh giá được được độ chính xác của từng khối địa chính khác nhau.

- Để cải thiện được dữ liệu như trên, cần phân biệt được các khối địa chính có thông tin về tỷ lệ đo vẽ, loại đất đo vẽ, công nghệ đo vẽ, quy phạm tham chiếu, khu vực số hóa hay đo vẽ dạng số trực tiếp, hệ tọa độ... từ đó tiến hành cải thiện độ chính xác từng khối độc lập, sau đó tiếp ghép dữ liệu toàn xã/phường và tiến hành nắn chuyển và cải thiện độ chính xác dữ liệu của toàn $\mathrm{xã} /$ phường.

Để tự động trong quy trình cải thiện độ chính cho đề tài đã xây dựng được một Modul phần mềm VPAI chạy trên ứng dụng của phần mềm MicroStationV8i, với chức năng: nắn chuyển bản đồ địa chính cũ theo các điểm khống chế song trùng đảm bảo cải thiện theo 3 bước: cải thiện độ chính xác trong mỗi khối địa chính; cải thiện chất lượng tiếp ghép các khối địa chính; hiệu chỉnh dữ liệu tổng thể của xã/phường. Phần mềm có thể trích xuất và biên tập dữ liệu địa chính theo mảnh bản đồ sau khi cải thiện độ chính xác.

\subsection{Quy trình cải thiện độ chính xác của dũ liệu địa chính cũ.}

Bao gồm các bước được đề xuất như sau:

1) Kết nối dũ liệu làm việc (dũ liệu không gian và dĩ liệu thuộc tính): Dữ liệu ban đầu được đo vẽ hoặc số hóa theo mảnh bản đồ và giao nộp cho cơ quan quản lý ở định dạng *.dgn, các mảnh bản đồ địa chính được tổng hợp thành 1 file cho toàn xã/phường hoặc tổng hợp theo các khu vực có tỷ lệ khác nhau. Kỹ thuật viên cần tổng hợp toàn xã/phường và kiểm tra lại, rà soát các lỗi và chạy Topo. Dữ liệu này thường được quản lý và biên tập trong phần mềm Famis. Trong phần mềm VPAI của đề tài sẽ thiết kế công cụ nhận dữ liệu này và quản lý các đối tượng không gian, kèm theo thuộc tính của thửa đất.

2) Thiết lập đơn vị hành chính làm việc (mã $x \tilde{a}$, huyện, tỉnh): Dữ liệu địa chính được quản lý theo không gian và thuộc tính theo từng đơn vị hành chính xã/phường - quận/huyện - tỉnh/thành phố. Mỗi xã/phường đều có mã theoquy định chung của toàn tỉnh và cả nước. Trong phần mềm VPAI của đề tài sẽ thiết kế công cụ thiết lập đơn vị hành chính cho dữ liệu của xã/phương cần xử lý.

3) Phân khối dũ liệu: Khối dữ liệu địa chính trong mỗi phường sẽ được các kỹ thuật viên khoanh vẽ trực tiếp trên file *dgn. Mỗi khối sẽ có một thông tin thuộc tính khác nhau, bao gồm: Tỷ lệ đo vẽ; Thời điểm đo vẽ; Công nghệ đo vẽ; Số hóa/hoặc dữ liệu gốc; Đơn vị đo vẽ; Với sự khác biệt theo 5 thuộc tính trên giữa các khối sẽ tạo ra độ chính xác khác nhau, vì vậy công cụ phân khối sẽ phục vụ cho việc nắn chuyển từng khối riêng biệt. Tùy thuộc vào mỗi khối để thiết kế các điểm song trùng cho phù hợp đảm bảo cải thiện độ chính xác tốt nhất. Chức năng phân khối dữ liệu cho phép tạo ra các khối với ranh giới chạy theo các cạnh thửa hoặc đối tượng hình tuyến trên bản đồ địa chính. Trong phần mềm VPAI của đề tài sẽ thiết kế công cụ quản lý các khối này theo ký hiệu (mã khối).

4) Chọn điểm nắn song trùng cho tùng khối: Các điểm khống chế song trùng sau khi được thiết kế bố trí cho phù hợp với yêu cầu và theo từng khối sẽ được tính toán bình sai và tổng hợp ở file *.txt hoặc *.xls và được nhập vào phần mềm. Các điểm này sẽ được quản lý theo 3 loại điểm: điểm phục vụ nắn chuyển các khối, ký hiệu là CP-K1, CP-K2, CP-K3...; các điểm dùng để kiểm tra ký hiệu là KT-1; KT-2, KT-3...; các điểm dùng để nắn bình sai toàn xã/phường ký hiệu là CP-T1, CP-T2, CP-T3... Các điểm này đã được thiết kế từ ban đầu và quản lý trong 
VPAI ở 3 lớp dữ liệu.

5) Bình sai khối địa chính: Việc nắn chuyển dữ liệu của từng khối theo các điểm khống chế song trùng dựa trên thuật toán số bình phương nhỏ nhất để xác định các tham số chuyển đồi chung cho dữ liệu của toàn khối. Từ các tham số tính chuyển để tính cho tất cả các giá trị $\left(\mathrm{x}_{\mathrm{i}} \mathrm{y}_{\mathrm{i}}\right)$ của các thửa. Trong phần mềm VPAI của đề tài sẽ thiết kế công cụ tính các tham số chuyển đổi, bình sai dữ liệu và xuất dữ liệu theo các giá trị đỉnh thửa mới. Kết quả của chức năng bình sai khối sẽ cho ta một khối mới bao gồm các thửa đã được nắn và chuyển đổi. Quá trình bình sai khối được thực hiện lần lượt cho tất cả các khối trong phạm vi của xã/phường.

6) Tiếp ghép dĩ liệu các khối theo phuong pháp bình sai điều kiện: Các khối địa chính trong mỗi phường sau bình sai sẽ có sự biến động về vị trí, diện tích, dẫn đến việc tiếp ghép dữ liệu giữa các khối cần phải được chuẩn hóa lại, chính vì vậy phần mềm VPAI của đề tài cần có chức năng tự động tiếp ghép dữ liệu theo nguyên tắc khối đo vẽ ở tỷ lệ nhỏ phải tiếp vào khối có tỷ lệ lớn, theo phương pháp bình sai điều kiện, lấy cạnh hoặc diện tích của thửa đất trên khối tỷ lớn làm điều kiện tính. Nếu các khối cùng tỷ lệ nhưng phương pháp thành lập khác nhau sẽ ưu tiên khu vực thành lập bằng phương pháp toàn đạc làm điều kiện. Tương tự khu đo có độ chính xác cao hơn sẽ làm điều kiện cho các khối có độ chính xác thấp hơn. Trong phần mềm VPAI của đề tài sẽ thiết kế công cụ xử lý và tiếp ghép dữ liệu giữa các khối.

7) Xử lý lồi hình học của dũ liệu sau bình sai điều kiện: Sau bình sai điều kiện sẽ có những thửa tự động chuẩn hóa tiếp ghép, tuy nhiên có những thửa không đảm bảo hạn sai trong tiếp ghép cần được xử lý trực tiếp bằng kỹ thuật viên. Công việc này cần được rà soát và kiểm tra ngoại nghiệp trước khi quyết định xử lý tiếp biên trên file dữ liệu. Trong phần mềm VPAI cần thiết kế công cụ để xử lý các đối tượng hình học như bắt điểm, trùng điểm, chọn đối tượng...
8) Nắn và chuyển đổi dĩ liệu toàn

xã/phường: Toàn bộ dữ liệu địa chính của toàn xã/phường cần được xử lý bình sai bước 2 để đảm bảo dữ liệu về gần nhất với vị trí thực trên thực địa. Việc nắn chuyển dữ liệu của cả xã/phường dựa theo các điểm khống chế song trùng bằng thuật toán số bình phương nhỏ nhất để xác định các tham số chuyển đổi chung cho toàn bộ dữ liệu của xã/phường. Từ các tham số tính chuyển để tính cho tất cả các giá trị $\left(\mathrm{x}_{\mathrm{i}} \mathrm{y}_{\mathrm{i}}\right)$ của các thửa. Trong phần mềm VPAI của đề tài sẽ thiết kế công cụ tính các tham số chuyển đổi, bình sai dữ liệu và xuất dữ liệu theo các giá trị đỉnh thửa mới. Kết quả của chức năng nắn chuyển sẽ cho ta một file dữ liệu mới bao gồm các thửa đã được nắn và chuyển đổi.

9) Tạo lại vùng toàn xã/phưòng: Sau khi dữ liệu toàn xã/phường đã được tiếp ghép và xử lý hoàn chỉnh, sẽ được biên tập lại bao gồm việc chạy kiểm tra lỗi, các lỗi như hở, trùng, bắt chưa tới, bắt vượt quá... trong phần mềm VPAI sẽ thiết kế chức năng kiểm tra dữ liệu. Sau đó sẽ chạy Topo lại toàn xã/phường, tính diện tích, tạo nhãn.

10) Phân mảnh cho dĩ liệu toàn xã/phuoòng: Chức năng phân mảnh theo quy định của Thông tư 25/2014/TT-BNTMT. Tạo khung bản đồ, bảng chắp, chú giải ngoài khung, bảng ghi chú thửa nhỏ... tên mảnh, số hiệu mảnh...

\subsection{Thục nghiệm cải thiện độ chính xác cho dũ liệu địa chính phwờng Khương Đình quận Thanh Xuân}

Bản đồ địa chính phường Khương Đình được thu thập tại phòng Tài nguyên và Môi trường quận Thanh Xuân. Tổng số mảnh bản đồ tỷ lệ 1:500 gồm 16 mảnh; tỷ lệ 1:1.000 gồm 3 mảnh. Thời gian đo vẽ tháng 12/1995, do Công ty Đo đạc địa chính Hà Nội đo vẽ và biên tập. Bản đồ được thành lập trong hệ tọa độ HN72, kinh tuyến trục $105^{\circ}$. Toàn bộ 19 mảnh bản đồ đã được số hóa và lưu ở khuôn dạng *.dgn

Các điểm song trùng sử dụng để nắn chuyển cải thiện độ chính xác bản đồ địa chính được đo bằng công nghệ GNSS-CORS bằng tín hiệu cải chính Reatime từ trạm CORS trong mạng lưới 


\section{VNGEONET.}

Nhóm thực hiện đề tài đã tiến hành đối soát 19 mảnh bản đồ địa chính tại thực địa kết quả cho thấy mức độ biến động rất lớn. Chủ yếu đất nông nghiệp đã chuyển đồi thành đất ở, đất công trình. Mức độ biến động đến trên $50 \%$.

Cho đến nay hơn 20 năm sử dụng dữ liệu này trong công tác quản lý. Phường Khương Trung, quận Thanh Xuận vẫn tiếp tục cập nhật, chỉnh lý trực tiếp trên file bản đồ số chạy trên phần mềm MicroStation. Dũ liệu đã được chuyển về hệ VN2000. Tại thời điểm này Hà Nội đang đo đạc chỉnh lý đồng bộ dữ liệu trên toàn Quận trực tiếp trên nền dữ liệu này. Do vậy việc cải thiện độ chính xác của 19 mảnh bản đồ này là cần thiết cho thực tế tại địa phương.

Qua kiểm tra và đánh giá chất lượng của bản đồ gốc tại 7 điểm kiểm tra trên tỷ lệ 1:500, sai số trung phương vị trí điểm của 15 điểm góc thửa là $17,7 \mathrm{~cm}$ vượt quá yêu cầu hiện nay là $10 \mathrm{~cm}$. Kiểm tra và đánh giá chất lượng của bản đồ gốc tại 8 điểm kiểm tra trên tỷ lệ $1: 1.000$, sai số trung phương vị trí điểm của 10 điểm góc thửa là $23,5 \mathrm{~cm}$ vượt quá yêu cầu hiện nay là $20 \mathrm{~cm}$.

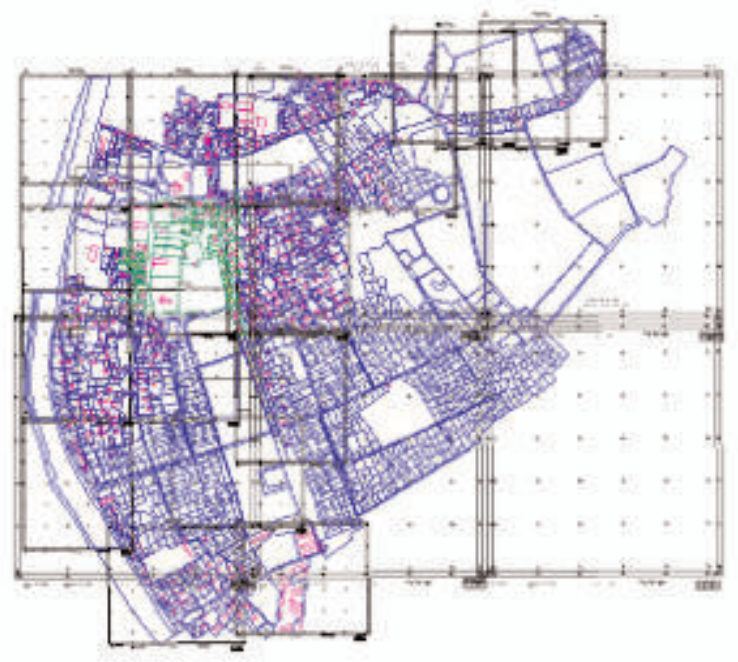

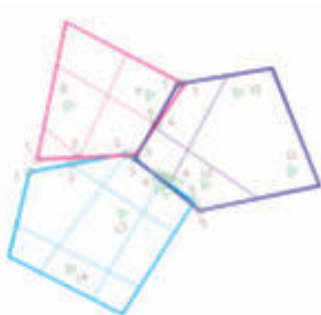
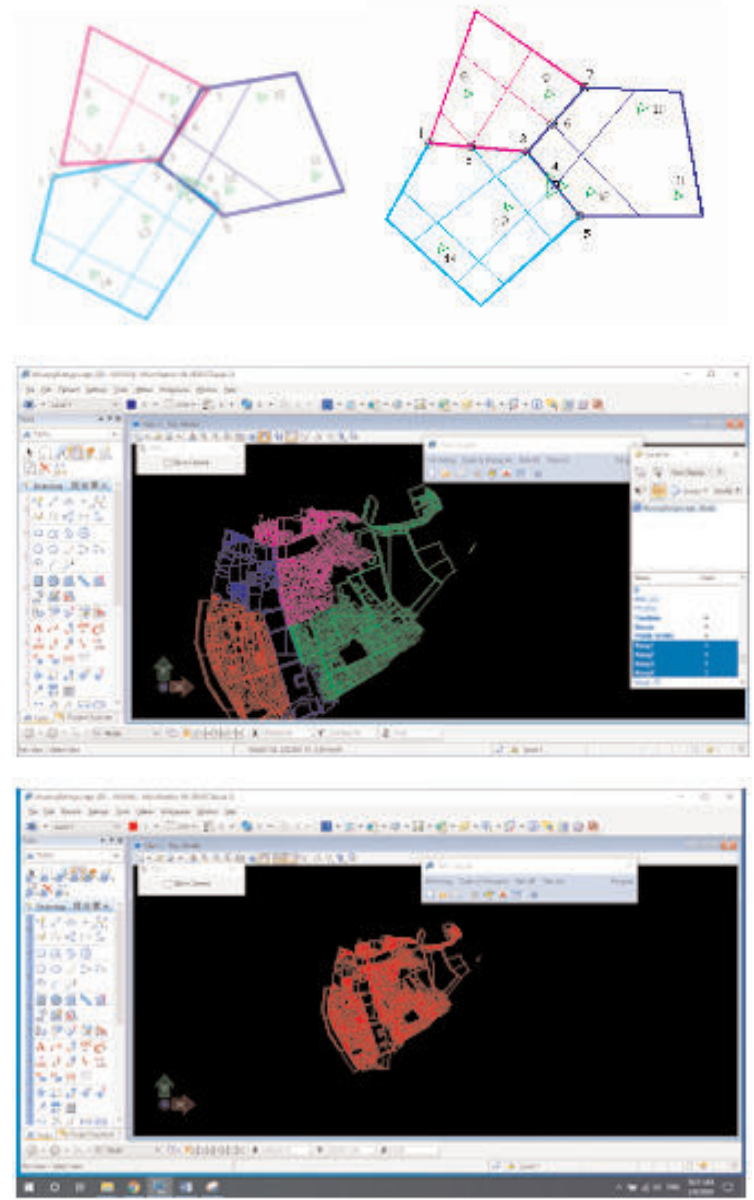

Hình 2: Minh họa các bước trong phần mềm VPAI cho dũ liệu địa chính phường Khuoong Đìn

Chọn điểm nắn cho 4 khối địa chính và điểm song trùng là góc thửa trên bản đồ. Mỗi khối địa chính có 5 điểm khống chế song trùng để cải thiện độ chính xác.

Công việc tiếp biên giữa các khối theo nguyên tắc: Thứ nhất, dựa vào các điểm xong trùng tính toán nắn chuyển khối cần tiếp biên theo khối dữ liệu có độ chính xác cao hơn (hoặc khối có tỷ lệ đo vẽ lớn hơn, tức là lấy khối có độ chính xác cao hơn làm chuẩn để đưa khối có độ chính xác kém hơn tiếp biên vào) theo phương pháp Helmet; Thứ hai, sau khi nắn chuyển về các tọa độ bị thay đổi dẫn đến diện tích và chiều dài các cạnh của các thửa biên bị thay đổi, vì vậy các 
thửa này sẽ được bình sai theo các điều kiện để phân bổ dần các sai số cho các đỉnh thửa lân cận tiếp theo, với yêu cầu phải nằm trong sai số vị trí cho phép.

Tổng hợp 4 lớp dữ liệu của 4 khối (Khoi1_adj, Khoi2_adj, Khoi3_adj, Khoi4_adj) tiến hành thực hiện chức năng chọn điểm nắn phân bố trên toàn xã/phường. Nắn toàn bộ dữ liệu xã/phường theo các điểm song trùng: Đề tài đã sử dụng 8 điểm khống chế song trùng để cải thiện độ chính xác đối với dữ liệu địa chính toàn phường Khương Đình.

Cuối cùng sử dụng 12 điểm khống chế đã đo GNSS-CORS để kiểm tra tại 5 điểm góc thửa còn tồn tại trên thực địa (trong đó 7 điểm ở tỷ lệ $1: 500$ và 5 điểm ở tỷ lệ $1: 1.000)$ chất lượng đã nâng nâng lên, đối với 1:500 sai số trung bình đạt $7,1 \mathrm{~cm}$ và $1: 1.000$ là $18,0 \mathrm{~cm}$. Chất lượng này hoàn toàn phù hợp với yêu cầu kỹ thuật đo vẽ bản đồ địa chính quy định tại Thông tư 25/2017/TT-BTNMT hiện nay.

\section{Kết luận}

Kết quả thực nghiệm của đề tài khi triển khai cải thiện độ chính xác đối với dữ liệu địa chính của phường Khương Đình cho thấy: từ dữ liệu địa chính được đo vẽ từ năm 1988 bằng thiết bị toàn đạc phổ thông, bản đồ gốc được số hóa và lưu trữ ở khuôn dạng *.dgn, bản đồ được đo vẽ ở 2 tỷ lệ $1: 500$ cho đất thổ cư và 1:1.000 cho đất nông nghiệp. Qua kiểm tra và đánh giá chất lượng của bản đồ gốc tại 7 điểm kiểm tra trên tỷ lệ 1:500, sai số trung phương vị trí điểm của 15 điểm góc thửa là $17,7 \mathrm{~cm}$ vượt quá yêu cầu hiện nay là $10 \mathrm{~cm}$. Kiểm tra và đánh giá chất lượng của bản đồ gốc tại 8 điểm kiểm tra trên tỷ lệ $1: 1.000$, sai số trung phương vị trí điểm của 10 điểm góc thửa là $23,5 \mathrm{~cm}$ vượt quá yêu cầu hiện nay là $20 \mathrm{~cm}$. Đề tài đã sử dụng 25 điểm khống chế song trùng để cải thiện độ chính xác đối với dữ liệu địa chính Khương Đình, kết quả sau kiểm tra tại 12 điểm (trong đó 7 điểm ở tỷ lệ $1: 500$ và 5 điểm ở tỷ lệ $1: 1.000)$ chất lượng đã nâng nâng lên, đối với 1:500 sai số trung bình đạt $7,1 \mathrm{~cm}$ và $1: 1.000$ là $18 \mathrm{~cm}$. Chất lượng này hoàn toàn phù hợp với yêu cầu kỹ thuật đo vẽ bản đồ địa chính quy định tại Thông tư 25/2017/TT-BTNMT.O

\section{Tài liệu tham khảo}

[1]. Felus, Y. A. (2007). On the Positional Enhancement of Digital Cadastral Maps. Survey Review, 39(306), 268-281. doi: 10.1179/175227007x197183.

[2]. Hesse, W. J., Benwell, G. L., \& Williamson, I. P. (1990). Optimising, maintaining and updating the spatial accuracy of digital cadastral data bases. Australian surveyor, 35(2), 109- 119.

[3]. Hope, S., Gordini, C., \& Kealy, A. (2008). Positional accuracy improvement: lessons learned from regional Victoria, Australia. Survey Review, 40(307), 29-42. doi: $10.1179 / 003962608 \times 253457$.

[4]. Frank Gielsdorf. 2004. Positional Accuracy Improvement - A Necessary Tool for Updating and Integration of GIS Data. TS2.2 Positional Accuracy Improvement - A Necessary Tool for Updating and Integration of GIS Data. FIG Working Week 2004. Athens, Greece, Mai 22-27, 2004.

[5]. Hashim et al. 2016. CADASTRAL POSITIONING ACCURACY IMPROVEMENT: A CASE STUDY IN MALAYSIA. The International Archives of the Photogrammetry, Remote Sensing and Spatial Information Sciences, Volume XLII-4/W1, 2016 International Conference on Geomatic and Geospatial Technology (GGT) 2016, 3-5 October 2016, Kuala Lumpur, Malaysia.

[6]. Nedim Tuno; Admir Mulahusić;

Dušan Kogoj (2017) Improving the Positional Accuracy of Digital Cadastral Maps through Optimal Geometric Transformation. Journal of Surveying Engineering/Volume 143 Issue 3 - August 2017. ASCE.

(Xem tiếp trang 55) 